Article

\title{
Preparation of Salvianolic Acid B Disodium Salt Considering the Water Extract Quality Standard
}

\author{
Tiantian Ye, Haibin Qu and Xingchu Gong * \\ Pharmaceutical Informatics Institute, College of Pharmaceutical Sciences, Zhejiang University, \\ Hangzhou 310058, China; 11319023@zju.edu.cn (T.Y.); quhb@zju.edu.cn (H.Q.) \\ * Correspondence: gongxingchu@zju.edu.cn; Tel./Fax: +86-571-8820-8426
}

Received: 6 March 2019; Accepted: 28 March 2019; Published: 1 April 2019

\begin{abstract}
A preparation process of salvianolic acid B (SAB) disodium salt from Salvia miltiorrhiza Bunge (Danshen) is provided in this work. A water extract quality standard was also developed to estimate the influences of Danshen quality on SAB disodium salt quality at an early stage of the preparation process. Crude SAB solution was obtained after water extraction, concentration, acidification, 1-butanol extraction, water washing, basification, and water back extraction. Extraction temperature, extraction $\mathrm{pH}$, and back-extraction $\mathrm{pH}$ were identified to be key parameters for the preparation of crude SAB solution. These parameters were optimized with Box-Behnken designed experiments. Crude SAB solution was further purified with a chromatography process. AMBERCHROW CG161M resin was selected as the best adsorbent. SAB disodium salt could be obtained by drying the eluate. Considering the quality of Danshen may affect the purity and yield of SAB disodium salt, different batches of Danshen were used to prepare SAB disodium salt with the optimized parameters. Water extract indices of phenolic compound purity and phenolic compound yield were measured. By developing models between SAB disodium salt purity and yield with water extract indices, the quality standard of Danshen water extract was obtained. The application of water extract quality standards can improve the quality consistency of SAB disodium salt. The effects of different batches of Danshen raw materials on the final product could be evaluated at the beginning of production stages. The present method could prepare about five grams of high-purity SAB disodium salt $(>95 \%)$ in one preparation cycle. The method reported in this work can also be used to develop process intermediate quality standards for other natural products.
\end{abstract}

Keywords: crude SAB preparation; quality standard of water extract; salvianolic acid B disodium salt; process optimization

\section{Introduction}

Danshen, the dried root of Salvia miltiorrhiza Bunge, is one of the most popular Chinese medicinal plants [1]. Phenolic acids and tanshinones are major active components in Danshen [2,3]. Salvianolic acid $\mathrm{B}(\mathrm{SAB})$ is the most abundant among the phenolic acids [4] with the bioactivities as an antioxidant [5,6], improving regional cerebral blood flow [7], protection against atherosclerosis [8], and so forth.

There are many publications on the purification of SAB from Danshen, with different technologies of liquid-liquid extraction [9], high-speed countercurrent chromatography [10-13], and resin chromatography [14-16]. Nie et al. [17] used ionic liquid-modified silica gel for the separation of water-soluble phenolic acids, including SAB. Guo et al. [18] reported a two-step procedure for the large-scale purification of $\mathrm{SAB}$ using an aqueous two-phase extraction followed by preparative high-performance liquid chromatography (HPLC). Li et al. [19] developed a method of online 
hyphenation of expanded bed adsorption chromatography and countercurrent chromatography for extraction and purification of SAB from Danshen.

The publications on SAB preparation focused on the development of preparation processes. However, the effect of Danshen quality was not considered. Zhong et al. [20] found that medicinal herb quality may show a greater impact on herbal product quality than that of the production process. The analysis of the chemical marker contents of a small amount of medicinal herbs is easy. However, medicinal herbs are heterogeneous. There may be a remarkable difference between the chemical marker contents obtained from a small sample analysis and the overall average of a large number of herbs. It indicates that accurate measurement of herb quality is not easy. An alternative method is to determine the chemical marker contents in the solvent extract of medicinal herbs, which is usually a homogeneous solution.

In this work, the preparation process of SAB disodium salt was developed and is shown in Figure 1. Crude SAB solution was prepared via water extraction, concentration, acidification, 1-butanol extraction, water washing, basification, and water back-extraction. After that, SAB disodium salt was obtained using a chromatography process and drying. Water extract was considered as a critical intermediate because its features reflect the impacts of Danshen materials and the water extraction process. If the water extract is not up to standard, there will be no need to carry out downstream purification processes.

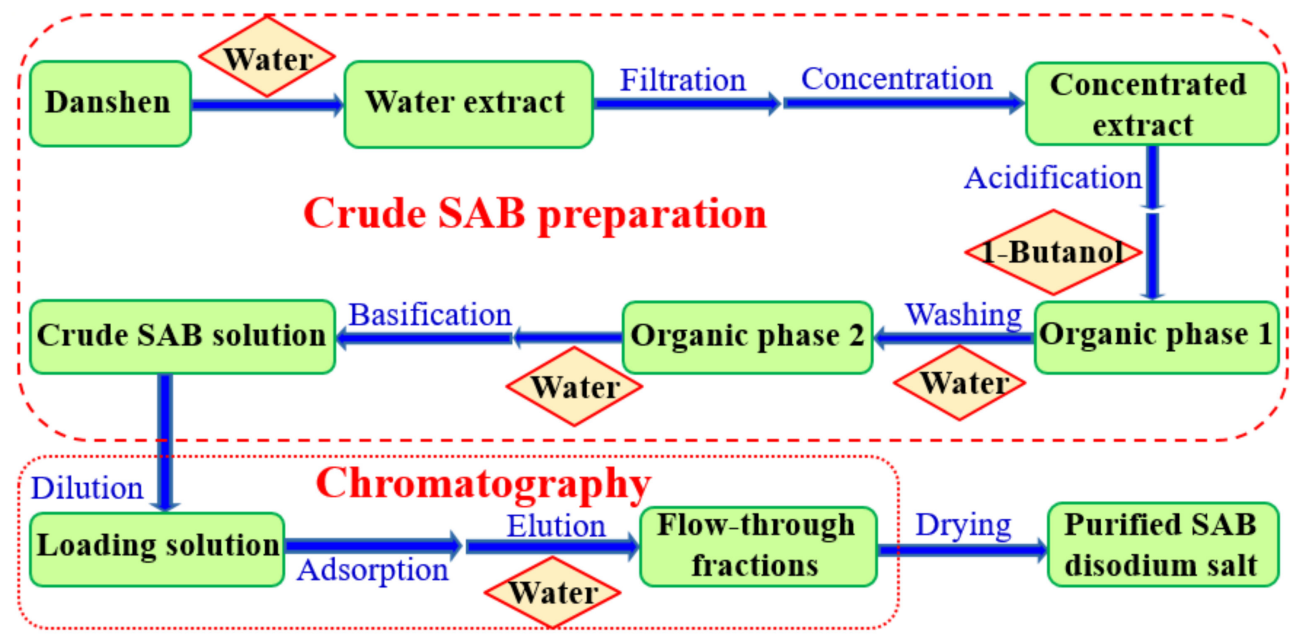

Figure 1. Schematic diagram of the preparation of SAB disodium salt.

In order to develop water extract standards, two procedures were carried out, as shown in Figure 2. First, process parameters were optimized with the same batch of Danshen herb. Second, different batches of Danshen herbs were processed with the optimized process parameters. The features of water extracts and SAB disodium salt products were determined. After modeling and calculation, the standard of water extracts was obtained. A verification experiment was carried out.

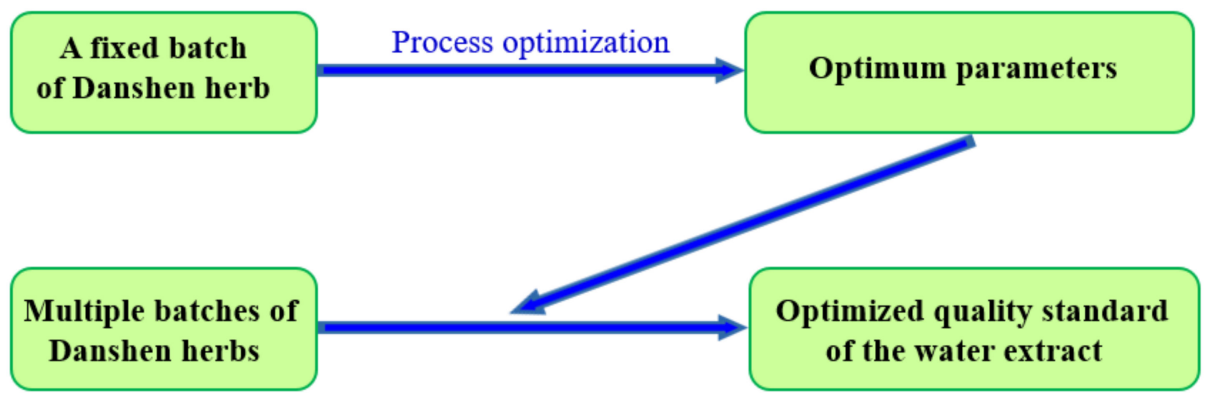

Figure 2. Flow chart of the development of the quality standard for a water extract. 


\section{Results and Discussion}

\subsection{Crude $S A B$ Preparation}

The Plackett-Burman designed experiments and results are presented in Table 1 . The results of partial regression coefficients, importance factors, and coefficient confidence intervals are listed in Table S1. The parameters with the importance factor $\left(I F_{j}\right.$, subscript $j$ refers to a process parameter) value ranked in the top three of all the seven parameters of crude SAB preparation process were selected as key parameters. Therefore, water extraction temperature, 1-butanol extraction $\mathrm{pH}$, and back-extraction $\mathrm{pH}$ were selected as key parameters of the crude SAB preparation process.

Table 1. Plackett-Burman design conditions and results.

\begin{tabular}{|c|c|c|c|c|c|c|c|c|c|}
\hline \multirow[b]{2}{*}{ Run } & \multicolumn{7}{|c|}{ Process Parameters } & \multicolumn{2}{|l|}{ Process Indices } \\
\hline & $\begin{array}{c}X_{1} \\
\left({ }^{\circ} \mathrm{C}\right)\end{array}$ & $X_{2}$ & $X_{3}$ & $\begin{array}{l}X_{4} \\
\text { (h) }\end{array}$ & $\begin{array}{c}X_{5} \\
(\mathrm{~mL} / \mathrm{g})\end{array}$ & $\begin{array}{c}X_{6} \\
(\mathrm{~mL})\end{array}$ & $\begin{array}{c}X_{7} \\
(\mathrm{~mL})\end{array}$ & $\begin{array}{c}Y_{1} \\
\text { (mg/g Medicinal Slices) }\end{array}$ & $\begin{array}{l}Y_{2} \\
(\%)\end{array}$ \\
\hline 1 & 50 & 4.2 & 5.0 & 2.0 & 8.0 & 60 & 100 & 0.516 & 37.7 \\
\hline 2 & 50 & 2.4 & 4.0 & 2.0 & 10.0 & 80 & 100 & 2.72 & 53.9 \\
\hline 3 & 100 & 2.4 & 5.0 & 4.0 & 10.0 & 60 & 50 & 9.23 & 38.7 \\
\hline 4 & 75 & 3.3 & 4.5 & 3.0 & 9.0 & 70 & 75 & 5.17 & 65.2 \\
\hline 5 & 50 & 2.4 & 4.0 & 2.0 & 8.0 & 60 & 50 & 2.69 & 57.2 \\
\hline 6 & 50 & 4.2 & 5.0 & 4.0 & 8.0 & 80 & 50 & 0.970 & 58.1 \\
\hline 7 & 100 & 2.4 & 5.0 & 2.0 & 8.0 & 80 & 50 & 10.2 & 49.0 \\
\hline 8 & 100 & 2.4 & 4.0 & 4.0 & 8.0 & 60 & 100 & 5.09 & 45.9 \\
\hline 9 & 75 & 3.3 & 4.5 & 3.0 & 9.0 & 70 & 75 & 5.33 & 63.8 \\
\hline 10 & 100 & 4.2 & 5.0 & 2.0 & 10.0 & 60 & 100 & 0.881 & 33.1 \\
\hline 11 & 50 & 2.4 & 5.0 & 4.0 & 10.0 & 80 & 100 & 10.8 & 64.2 \\
\hline 12 & 75 & 3.3 & 4.5 & 3.0 & 9.0 & 70 & 75 & 5.61 & 62.9 \\
\hline 13 & 100 & 4.2 & 4.0 & 2.0 & 10.0 & 80 & 50 & 0.300 & 38.8 \\
\hline 14 & 100 & 4.2 & 4.0 & 4.0 & 8.0 & 80 & 100 & 0.238 & 28.5 \\
\hline 15 & 50 & 4.2 & 4.0 & 4.0 & 10.0 & 60 & 50 & 0.205 & 39.5 \\
\hline
\end{tabular}

$\mathrm{X}_{1}$ : extraction temperature; $\mathrm{X}_{2}$ : extraction $\mathrm{pH} ; \mathrm{X}_{3}$ : back-extraction $\mathrm{pH} ; \mathrm{X}_{4}$ : extraction time; $\mathrm{X}_{5}$ : water consumption for extraction; $\mathrm{X}_{6}$ : 1-butanol amount; $\mathrm{X}_{7}$ : water consumption for washing; $\mathrm{Y}_{1}$ : $\mathrm{SAB}$ yield in crude $\mathrm{SAB}$ solution; $\mathrm{Y}_{2}$ : $\mathrm{SAB}$ purity in crude $\mathrm{SAB}$ solution.

Table 2 displays the results of Box-Behnken designed experiments. SAB yield in crude $\mathrm{SAB}$ solution $\left(S A B_{\text {yield }}^{S}\right.$, superscript $S$ refers to the crude $\mathrm{SAB}$ solution) was between 0.227 and $10.5 \mathrm{mg} / \mathrm{g}$ medicinal slices. SAB purity in crude $\mathrm{SAB}$ solution $\left(S A B_{\text {purity }}^{S}\right)$ varied from $43.5 \%$ to $59.9 \%$. The estimated parameter values, determination coefficients $\left(\mathrm{R}^{2}\right)$, ANOVA results, and coefficient confidence intervals of quadratic models are shown in Table S2. The values of $\mathrm{R}^{2}$ of the two models were higher than 0.85 . The two models were significant with $p$-values less than $0.01 . S A B_{\text {purity }}^{S}$ increased as extraction temperature decreased. 
Table 2. Box-Behnken design conditions and results.

\begin{tabular}{|c|c|c|c|c|c|}
\hline \multirow{2}{*}{ Run } & \multicolumn{3}{|c|}{ Key Parameters of Crude SAB Preparation Process } & \multicolumn{2}{|l|}{ Process Indices } \\
\hline & $X_{1}\left({ }^{\circ} \mathrm{C}\right)$ & $X_{2}$ & $X_{3}$ & $Y_{1}(\mathrm{mg} / \mathrm{g}$ Medicinal Slices) & $Y_{2}(\%)$ \\
\hline S1 & 100 & 3.3 & 4.0 & 1.59 & 47.0 \\
\hline S2 & 75 & 3.3 & 4.5 & 5.46 & 59.2 \\
\hline S3 & 75 & 2.4 & 5.0 & 10.5 & 54.1 \\
\hline S4 & 100 & 3.3 & 5.0 & 7.67 & 48.9 \\
\hline S5 & 50 & 2.4 & 4.5 & 5.28 & 53.6 \\
\hline S6 & 100 & 2.4 & 4.5 & 8.19 & 43.5 \\
\hline S7 & 100 & 4.2 & 4.5 & 0.741 & 50.2 \\
\hline S8 & 50 & 3.3 & 4.0 & 1.06 & 55.7 \\
\hline S9 & 50 & 4.2 & 4.5 & 0.506 & 52.1 \\
\hline S10 & 75 & 3.3 & 4.5 & 5.26 & 59.4 \\
\hline S11 & 75 & 4.2 & 4.0 & 0.227 & 46.8 \\
\hline S12 & 50 & 3.3 & 5.0 & 5.11 & 57.8 \\
\hline S13 & 75 & 2.4 & 4.0 & 2.72 & 43.9 \\
\hline S14 & 75 & 3.3 & 4.5 & 5.55 & 59.9 \\
\hline S15 & 75 & 4.2 & 5.0 & 1.09 & 44.2 \\
\hline S16 & 75 & 3.3 & 4.5 & 6.07 & 58.1 \\
\hline
\end{tabular}

The contour plots of the obtained mathematical models for the $S A B_{\text {yield }}^{S}$ and $S A B_{\text {purity }}^{S}$ are displayed in Figures 3 and 4. In Figure 3a-c, higher extraction temperature, higher back-extraction $\mathrm{pH}$, and lower extraction $\mathrm{pH}$ led to a higher $S A B_{\text {yield }}^{S}$, respectively.

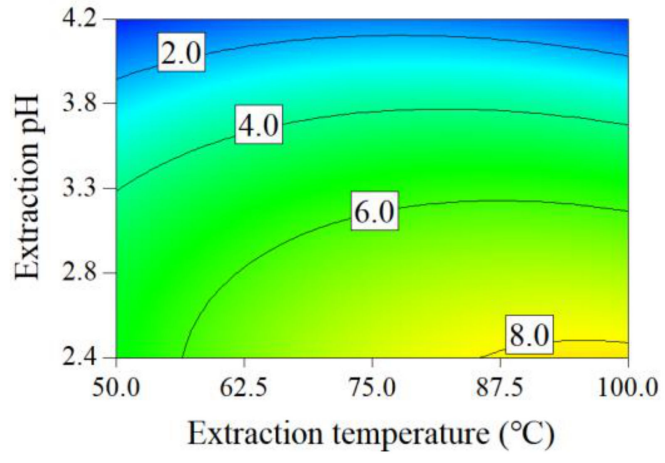

(a)

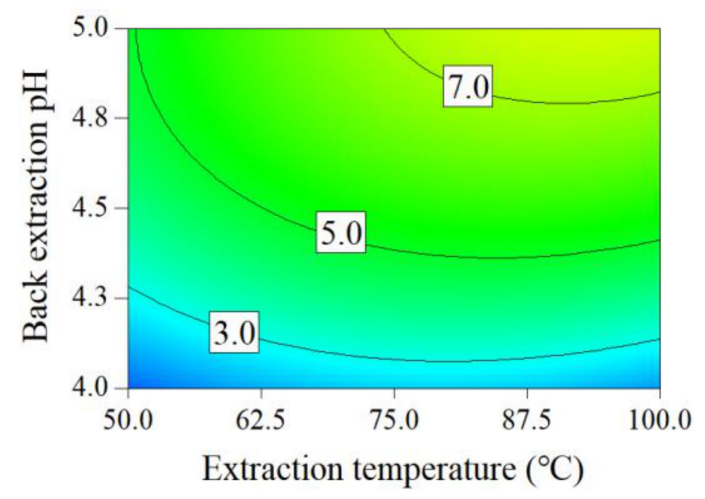

(b)

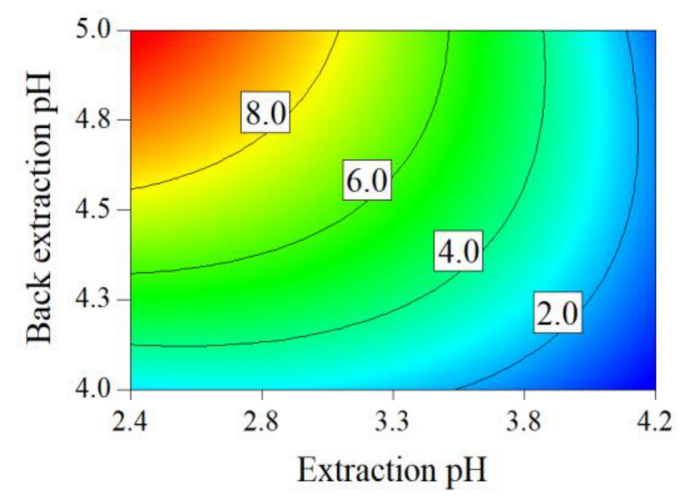

(c)

Figure 3. Contour plots for key process parameter effects on the $S A B_{\text {yield. }}^{S}$ (a) Extraction $\mathrm{pH}$ and extraction temperature effect on the $S A B_{\text {yield }}^{S}$ when back-extraction $\mathrm{pH}=4.5$, (b) back-extraction $\mathrm{pH}$ and extraction temperature effect on the $S A B_{\text {yield }}^{S}$ when extraction $\mathrm{pH}=3.3$, (c) back-extraction $\mathrm{pH}$ and extraction $\mathrm{pH}$ effect on the $S A B_{\text {yield }}^{S}$ when extraction temperature $=75.0^{\circ} \mathrm{C}$. 


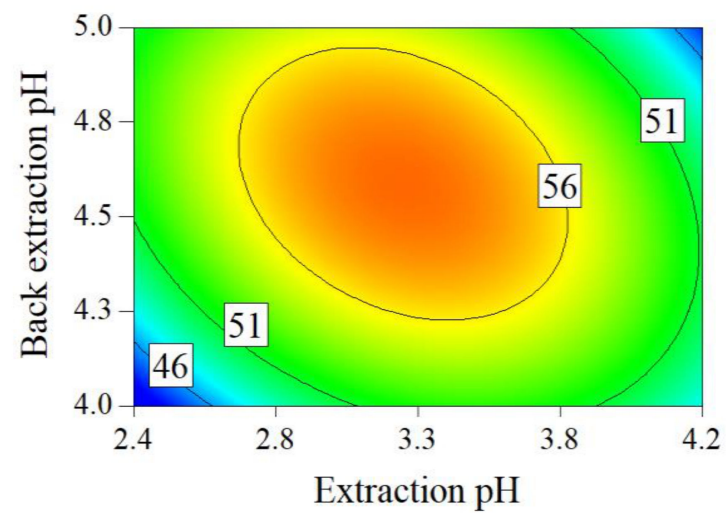

Figure 4. Contour plots for key process parameter effects on the $S A B_{\text {purity }}^{S}$ (extraction temperature = $\left.75.0^{\circ} \mathrm{C}\right)$.

The optimized parameter ranges for the crude $\mathrm{SAB}$ preparation process are as follows: the extraction temperature was from $60{ }^{\circ} \mathrm{C}$ to $80{ }^{\circ} \mathrm{C}$, the extraction $\mathrm{pH}$ was from 2.5 to 3.0, and the back-extraction $\mathrm{pH}$ was from 4.6 to 5.0. The $S A B_{\text {purity }}^{S}$ and $S A B_{\text {yield }}^{S}$ were more than $50.0 \%$ and higher than $5.0 \mathrm{mg} / \mathrm{g}$ medicinal slices under the above optimized parameters.

\subsection{SAB Purification with Chromatography Process}

\subsubsection{Resin Screening by Adsorption Capacity, Desorption Capacity, and Desorption Ratio}

The adsorption and desorption capacities of different resins for the SAB were shown in Figure 5. High adsorption capacity ( $>60 \mathrm{mg} / \mathrm{g}$ dry resin) were observed for the resins of FPA98Cl, FPA90Cl, and FPA53. However, the adsorbed SAB could hardly be desorbed with ethanol solution. The results were reasonable because these three resins are anion exchange resins. The adsorption capacities of polyamide, HPD100, and AB-8 resins were lower than $30 \mathrm{mg} / \mathrm{g}$ dry resin. The adsorption capacity of $50 \mathrm{~W} \times 2,50 \mathrm{~W} \times 4, \mathrm{CG} 161 \mathrm{M}$, and XAD $1600 \mathrm{~N}$ were higher than $30 \mathrm{mg} / \mathrm{g}$ dry resin. The desorption ratio of $50 \mathrm{~W} \times 2,50 \mathrm{~W} \times 4$, and CG161M were higher than $80 \%$. Therefore, $50 \mathrm{~W} \times 2,50 \mathrm{~W} \times 4$, and CG161M were chosen to be further investigated.

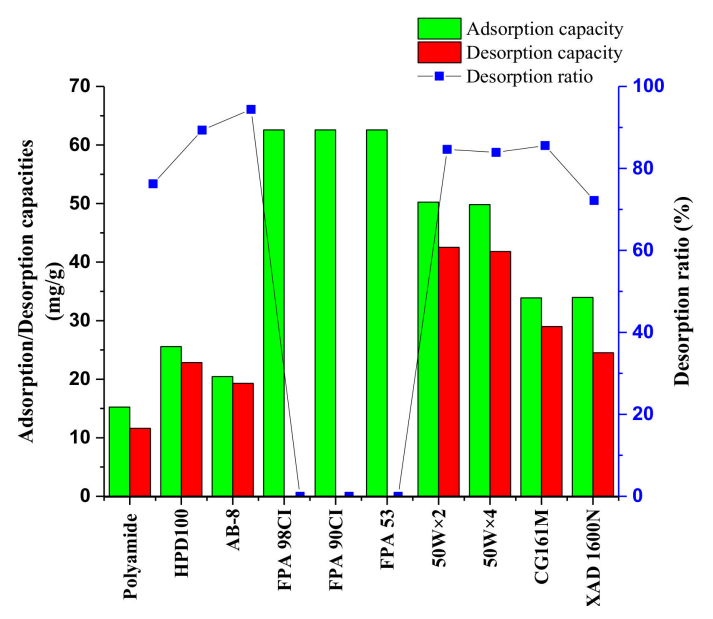

Figure 5. Adsorption and desorption capacities, and the desorption ratio of SAB on ten resins.

\subsubsection{Resin Screening by Adsorption Kinetics}

Adsorption kinetics of SAB were measured with CG161M, $50 \mathrm{~W} \times 2$, and $50 \mathrm{~W} \times 4$ resins at $30^{\circ} \mathrm{C}$. The results are shown in Figure 6. The adsorption amount of SAB increased with the adsorption time for all three resins. For resin CG161M, equilibrium was almost reached after $30 \mathrm{~min}$. For the other two resins, at least $120 \mathrm{~min}$ was required to reach equilibrium. 


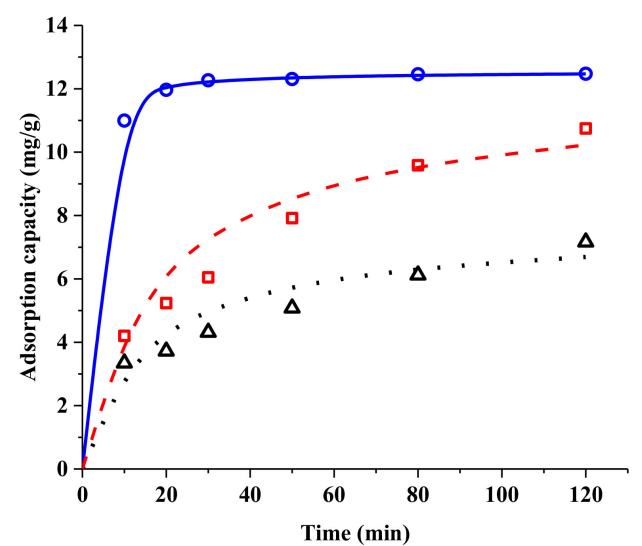

Figure 6. Adsorption kinetics of SAB fitted to pseudo-second-order model. Solid line, CG161M resin, (calc.); dashed line, $50 \mathrm{~W} \times 4$ resin, (calc.); dotted line, $50 \mathrm{~W} \times 2$ resin, (calc.); $\bigcirc$, CG161M resin, (exp.); $\square, 50 \mathrm{~W} \times 4$ resin, (exp.); $\triangle, 50 \mathrm{~W} \times 2$ resin, (exp.).

The pseudo-second-order model was used to fit the experimental results. In Table 3, all the $\mathrm{R}^{2}$ values are higher than 0.95 . The values of $k_{2}$ for CG161M, $50 \mathrm{~W} \times 2$, and $50 \mathrm{~W} \times 4$ resins were $0.103 \mathrm{~min}^{-1}, 0.00900 \mathrm{~min}^{-1}$, and $0.00479 \mathrm{~min}^{-1}$, respectively. The highest $k_{2}$ value for CG161M meant it had the fastest absorption rate among the three resins. Therefore, the CG161M resin was selected for the purification of SAB.

Table 3. Pseudo-second-order kinetics parameters of resins calculated on the basis of SAB.

\begin{tabular}{cccc}
\hline \multirow{2}{*}{$\operatorname{Resin}$} & \multicolumn{3}{c}{ Pseudo-Second-Order } \\
\cline { 2 - 4 } & $\boldsymbol{Q}_{\boldsymbol{e}}$ (cal) (mg/g Dry Resin) & $\left.\boldsymbol{k}_{\mathbf{2}} \mathbf{( m i n}^{\mathbf{- 1}}\right)$ & $\mathbf{R}^{\mathbf{2}}$ \\
\hline $\mathrm{CG} 161 \mathrm{M}$ & 12.55 & 0.103 & 0.99 \\
$50 \mathrm{~W} \times 2$ & 7.519 & 0.00900 & 0.96 \\
$50 \mathrm{~W} \times 4$ & 11.74 & 0.00479 & 0.95 \\
\hline
\end{tabular}

$Q_{e}$ : the adsorption capacity at equilibrium; $k_{2}$ : the rate constant of pseudo-second-order model.

\subsubsection{Chromatographic Process}

After loading the diluted crude SAB solution to the CG161M column, the column was eluted with 2 bed volume (BV) of deionized water, 2 BV of $10 \% v / v$ ethanol, 2 BV of $30 \% v / v$ ethanol, and 2 BV of $50 \% v / v$ ethanol sequentially. The flow-through elution fractions were collected. The concentrations of $\mathrm{SAB}$ in the elution fractions were analyzed and are shown in Figure 7.

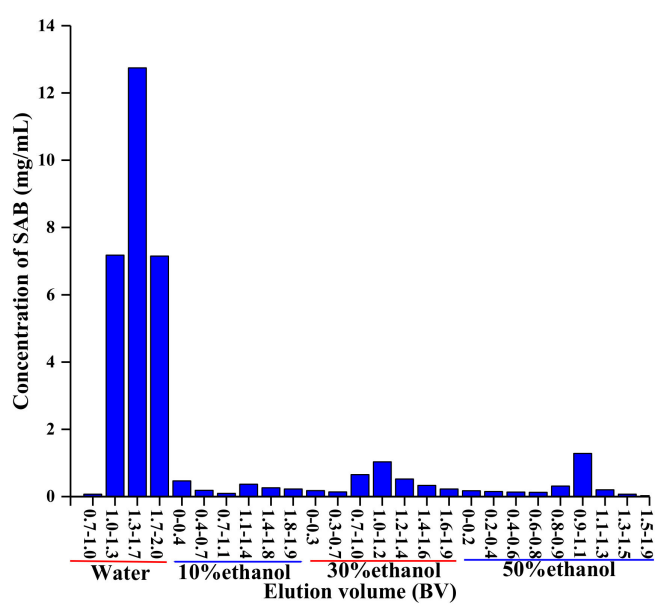

Figure 7. Elution profile of SAB on CG161M resin. 
More than $78.0 \% \mathrm{SAB}$ was collected from 1.0-2.0 BV water elution fractions. In contrast, only $14.6 \% \mathrm{SAB}$ was collected from $10 \%$ to $50 \%$ ethanol $(v / v)$ elution fractions. Most of the SAB was collected within water elution fractions. Thus, water was chosen as the desorption solvent for the purification of SAB. Elution volume of 1.0-2.0 BV was collected. The resin was regenerated with $90 \%$ ethanol $(v / v)$ for $8 \mathrm{BV}$ and water for $8 \mathrm{BV}$, respectively.

\subsection{Water Extract Standard}

The yield and purity of different phenolic compounds, including Danshensu (DSS), protocatechuic aldehyde (PA), caffeic acid (CA), rosmarinic acid (RA), lithospermic acid (LA), SAB, and salvianolic acid A (SAA), were used as quality indices of Danshen water extracts. The data are shown in Table 4. The corresponding values of the yield of $\mathrm{SAB}$ in final product $\left(S A B_{\text {yield }}^{F}\right)$ and the purity of $\mathrm{SAB}$ in final product $\left(S A B_{\text {purity }}^{F}\right)$ were also listed in Table 4. Stepwise regression was used to build the models between $S A B_{\text {yield }}^{F}$ and $S A B_{\text {purity }}^{F}$ data and water extract indices of phenolic compound purity $\left(P C_{\text {purity }}^{E}\right)$ and phenolic compound yield $\left(P C_{\text {yield }}^{E}\right)$. The $p$-values for adding and removing a term were set to 0.01. The Design Expert 10.0.4.0 software (Stat-Ease Inc., Minneapolis, MN, USA) was used for the calculations. $S A B_{\text {yield }}^{F}$ or $S A B_{\text {purity }}^{F}$ was assumed to be only affected by the phenolic compound yield or purity in water extracts. The obtained models were as follows:

$$
\begin{gathered}
S A B_{\text {purity }}^{F}=1.079-543.7 \times C A_{\text {purity }}^{E} R^{2}=0.9546 ; \\
S A B_{\text {yield }}^{F}=0.04756 \times S A B_{\text {yield }}^{E}-0.002992 R^{2}=0.7668
\end{gathered}
$$

where superscripts $E$ and $F$ refer to the water extract and the final product, respectively. The determination coefficients of regression Equations (1) and (2) were all higher than 0.76, which implied that most variations of experimental data can be explained.

In this work, a prediction error propagation method was used to estimate the water extract standards [21]. Taking Equation (1) as an example, the residuals were assumed to follow a normal distribution with a mean value of zero. The standard deviation of this normal distribution for the Equation (1) $\left(S D_{\text {Residual }}^{E q 1}\right)$ was assumed to be the same of that of residuals, which was 0.05575 . If $S A B_{\text {purity }}^{F}$ was expected to be not lower than $90 \%$ with a probability of $80 \%$, the following equation was used to calculate maximum $C A_{\text {purity }}^{E}$ value.

$$
S A B_{\text {purity }}^{F}=1.079-543.7 \times C A_{\text {purity }}^{E}-Z_{0.2} \times S D_{\text {Residual }}^{E q 1} \geq 0.900
$$

where $Z_{0.20}$ was the normal distribution boundary, which equaled 0.84 . Then, $C A_{\text {purity }}^{E}$ should be lower than $2.43 \times 10^{-4}$.

By using the same method, the standard deviation of the normal distribution for Equation (2) $\left(S D_{\text {Residual }}^{E q 2}\right)$ was assumed to be equivalent to that of the residuals, which was 0.1894 . If $S A B_{\text {yield }}^{F}$ was expected to be not lower than $0.300 \mathrm{mg} / \mathrm{g}$ medicinal slices with a probability of $80 \%$, the following equation was used to calculate the minimum $S A B_{\text {yield }}^{E}$ value:

$$
S A B_{\text {yield }}^{F}=0.04756 \times S A B_{\text {yield }}^{E}-0.002992-Z_{0.2} \times S D_{\text {Residual }}^{E q 2} \geq 0.300
$$

where $Z_{0.20}$ was the normal distribution boundary, which equaled 0.84 . Then, $S A B_{\text {yield }}^{E}$ should be higher than $9.72 \mathrm{mg} / \mathrm{g}$ medicinal slices. 
Table 4. The effects of yield and purity of phenolic compounds on $S A B_{\text {purity }}^{F}$ and $S A B_{\text {yield }}^{F}$.

\begin{tabular}{|c|c|c|c|c|c|c|c|c|c|c|c|c|c|c|c|c|c|}
\hline \multirow{2}{*}{ Run } & \multirow{2}{*}{ Habitat } & \multicolumn{7}{|c|}{ Phenolic Compounds Yield of Water Extract } & \multicolumn{7}{|c|}{ Phenolic Compounds Purity of Water Extract } & \multicolumn{2}{|c|}{ Final Product Indices } \\
\hline & & $\begin{array}{c}D S S_{\text {yield }}^{E} \\
(\mathrm{mg} / \mathrm{g} \\
\text { medicinal } \\
\text { slices) }\end{array}$ & $\begin{array}{c}P A_{\text {yield }}^{E} \\
\left(\times 10^{-2} \mathbf{m g} / \mathbf{g}\right. \\
\text { medicinal } \\
\text { slices })\end{array}$ & $\begin{array}{c}C A_{\text {yield }}^{E} \\
\left(\times 10^{-2} \mathrm{mg} / \mathrm{g}\right. \\
\text { medicinal } \\
\text { slices })\end{array}$ & $\begin{array}{c}R A_{\text {yield }}^{E} \\
(\mathrm{mg} / \mathrm{g} \\
\text { medicinal } \\
\text { slices })\end{array}$ & $\begin{array}{c}L A_{\text {yield }}^{E} \\
\text { (mg/g } \\
\text { medicinal } \\
\text { slices) }\end{array}$ & $\begin{array}{c}S A B_{\text {yield }}^{E} \\
\text { (mg/g } \\
\text { medicinal } \\
\text { slices) }\end{array}$ & $\begin{array}{c}S A A_{\text {yield }}^{E} \\
\text { (mg/g } \\
\text { medicinal } \\
\text { slices) }\end{array}$ & $\begin{array}{c}D S S_{\text {purity }}^{E} \\
\left(\times 10^{-2}, \%\right)\end{array}$ & $\begin{array}{c}P A_{\text {purity }}^{E} \\
\left(\times 10^{-2}, \%\right)\end{array}$ & $\begin{array}{c}C A_{\text {purity }}^{E} \\
\left(\times 10^{-2}, \%\right)\end{array}$ & $\begin{array}{c}R A_{\text {purity }}^{E} \\
(\%)\end{array}$ & $\begin{array}{c}L A_{\text {purity }}^{E} \\
(\%)\end{array}$ & $\begin{array}{c}S A B_{\text {purity }}^{E} \\
(\%)\end{array}$ & $\begin{array}{c}S A A_{\text {purity }}^{E} \\
(\%)\end{array}$ & $\begin{array}{c}S A B_{\text {purity }}^{F} \\
(\%)\end{array}$ & $\begin{array}{c}S A B_{\text {yield }}^{F} \\
(\mathrm{mg} / \mathrm{g} \\
\text { medicinal } \\
\text { slices) }\end{array}$ \\
\hline I & $\begin{array}{l}\text { Bozhou, } \\
\text { Anhui } \\
\text { Province }\end{array}$ & 0.295 & 1.08 & 5.54 & 1.15 & 0.685 & 22.6 & 0.301 & 9.81 & 0.358 & 1.84 & 0.381 & 0.228 & 7.53 & 0.100 & 95.9 & 1.42 \\
\hline III & $\begin{array}{l}\text { Minxian, } \\
\text { Gansu } \\
\text { Province }\end{array}$ & 0.428 & 2.54 & 27.9 & 1.34 & 0.0746 & 0.809 & 0.0322 & 23.7 & 1.41 & 15.4 & 0.742 & 0.0413 & 0.447 & 0.0178 & 22.5 & 0.00483 \\
\hline III & $\begin{array}{l}\text { Wanrong, } \\
\text { Shanxi } \\
\text { Province }\end{array}$ & 0.252 & 2.61 & 8.30 & 0.428 & 0.707 & 21.1 & 0.392 & 7.00 & 0.725 & 2.31 & 0.119 & 0.196 & 5.87 & 0.109 & 97.5 & 0.717 \\
\hline IV & $\begin{array}{c}\text { Dancheng, } \\
\text { Henan } \\
\text { Province }\end{array}$ & 0.377 & 1.92 & 6.40 & 0.709 & 0.976 & 20.1 & 0.372 & 12.1 & 0.617 & 2.05 & 0.228 & 0.313 & 6.45 & 0.119 & 88.3 & 0.784 \\
\hline $\mathrm{V}$ & $\begin{array}{l}\text { Zhongjiang, } \\
\text { Sichuan } \\
\text { Province }\end{array}$ & 0.310 & 1.83 & 7.35 & 0.452 & 0.845 & 11.2 & 0.230 & 15.7 & 0.928 & 3.72 & 0.229 & 0.428 & 5.66 & 0.116 & 99.2 & 0.659 \\
\hline VI & $\begin{array}{l}\text { Wanrong, } \\
\text { Shanxi } \\
\text { Province (1) }\end{array}$ & 0.359 & 0.923 & 6.43 & 0.238 & 0.800 & 11.3 & 0.132 & 10.7 & 0.275 & 1.92 & 0.0710 & 0.238 & 3.38 & 0.0393 & 96.0 & 0.559 \\
\hline VII & $\begin{array}{l}\text { Wanrong, } \\
\text { Shanxi } \\
\text { Province (2) }\end{array}$ & 0.322 & 0.842 & 6.20 & 0.231 & 0.781 & 11.1 & 0.128 & 9.83 & 0.257 & 1.89 & 0.0704 & 0.238 & 3.38 & 0.0391 & 96.7 & 0.492 \\
\hline VIII & $\begin{array}{c}\text { Wanrong, } \\
\text { Shanxi } \\
\text { Province (3) }\end{array}$ & 0.333 & 0.904 & 6.50 & 0.249 & 0.821 & 11.6 & 0.136 & 10.0 & 0.271 & 1.95 & 0.0746 & 0.246 & 3.50 & 0.0409 & 98.1 & 0.564 \\
\hline
\end{tabular}

DSS: Danshensu; PA: protocatechuic aldehyde; CA: caffeic acid; RA: rosmarinic acid; LA: lithospermic acid; SAA: salvianolic acid A. 


\subsection{Verification}

Verification experiments are displayed in Table 5. The conditions of the verification experiments were the same as the mentioned conditions in Section 3.4. The experimental value of $S A B_{\text {purity }}^{F}$ was $99.3 \%$ and agreed well with the prediction value. The experimental value of $S A B_{\text {yield }}^{F}$ was higher than $0.700 \mathrm{mg} / \mathrm{g}$ medicinal slices. These results indicated that the quality standards of Danshen water extract were reliable.

Table 5. The effects of $S A B_{\text {yield }}^{E}$ on $S A B_{\text {yield }}^{F}$ and $C A_{\text {purity }}^{E}$ on $S A B_{\text {purity }}^{F}$.

\begin{tabular}{ccccc}
\hline Habitat & $\begin{array}{c}S A B_{\text {yield }}^{E}(\mathrm{mg} / \mathrm{g} \\
\text { Medicinal Slices })\end{array}$ & $\begin{array}{c}C A_{\text {purity }}^{E} \\
\left(\times \mathbf{1 0}^{-2}, \mathbf{\%}\right)\end{array}$ & $S A B_{\text {purity }}^{F} / P V(\%)$ & $\begin{array}{c}S A B_{\text {yield }}^{F} / P V(\mathrm{mg} / \mathrm{g} \\
\text { Medicinal Slices) }\end{array}$ \\
\hline Julu, Hebei Province & $10.30 \quad$ & 2.31 & $99.3 / 95.3$ & $0.732 / 0.487$ \\
\hline \multicolumn{5}{c}{ PV predicted values. }
\end{tabular}

\subsection{Identification of SAB Disodium Salt}

After drying, a tan amorphous powder was obtained. The Fourier transform infrared spectroscopy (FTIR) measurements were carried out with a Fourier transform infrared spectrophotometer (JASCO, FT-IR4100, Tokyo, Japan) in the wavenumber range of 500-4000 $\mathrm{cm}^{-1}$. With the FTIR spectrum in Figure S1, the peak at $3423 \mathrm{~cm}^{-1}$ was assigned to the hydroxyl stretching vibration [22]. Compared to the carbonyl peaks of SAB $\left(1726 \mathrm{~cm}^{-1}, 1613 \mathrm{~cm}^{-1}, 1521 \mathrm{~cm}^{-1}\right.$, and $\left.1445 \mathrm{~cm}^{-1}\right)$, the four carbonyl peaks of this compound $\left(1719 \mathrm{~cm}^{-1}, 1590 \mathrm{~cm}^{-1}, 1520 \mathrm{~cm}^{-1}\right.$, and $\left.1440 \mathrm{~cm}^{-1}\right)$ with the low wavenumber shift may have been caused by the formation of the carboxylate. The absorption peaks of $1262 \mathrm{~cm}^{-1}$ and $1167 \mathrm{~cm}^{-1}$ were attributed to the characteristic vibration of the hydroxyl group on the benzene ring [22].

A Triple TOF 5600+ mass spectrometer (AB SCIEX, Framingham, MA, USA) provided useful information for the assessment of the cation, which is shown in Figure S2. The time-of-flight mass spectrometer (TOF-MS) fragments at $\mathrm{m} / \mathrm{z} 761$ of this compound suggested the presence of $[\mathrm{SAB}-\mathrm{H}]^{-}$ $(m / z 717)$ and two sodium ions. Hence, it was deduced that this powder might be a disodium salt of SAB.

An aqueous solution of the obtained powder was prepared at a concentration of $9.832 \mathrm{mg} / \mathrm{L}$. The $\mathrm{Na}^{+}$concentration of aqueous solution was then determined using an atomic absorption spectrophotometer (240AA, Agilent, PA, CA, USA). The concentration of sodium ions was $0.5955 \mathrm{mg} / \mathrm{L}$. If this powder was $\mathrm{SAB}$ disodium salt, the theoretical sodium ion concentration should be $0.5928 \mathrm{mg} / \mathrm{L}$, which is very close to the measured value.

Consequently, this powder was determined to be SAB disodium salt.

\section{Materials and Methods}

\subsection{Materials and Reagents}

Danshen (Salvia Miltiorrhiza) were originally collected from six different habitats in China (Bozhou, Anhui Province; Wanrong, Shanxi Province; Minxian, Gansu Province; Dancheng, Henan Province; Zhongiiang, Sichuan Province, and Julu, Hebei Province). Seven reference compounds, including sodium Danshensu (DSS-Na, lot. 180327, 98.92\%), PA (lot. 171126, 99.69\%), CA (lot. 180105, 99.62\%), RA (lot. 180403, 99.47\%), LA (lot. 180107, 98.85\%), SAB (lot. 180120, 99.36\%), and SAA (lot. 171229, 98.94\%) were purchased from Shanghai Winherb Medical Science (Shanghai, China) as standard compounds without further purification. HPLC-grade formic acid was obtained from ROE Scientific Inc. (Newark, DE, USA). HPLC-grade methanol and acetonitrile were purchased from Merck (Darmstadt, Germany). Analytical grade 1-butanol was supplied by Sinopharm Chemical Reagent (Beijing, China). Analytical grade ethanol was supplied by Shanghai Lingfeng Chemical 
Reagent (Shanghai, China). Analytical grade hydrochloric acid and sodium hydroxide were purchased from Sinopharm Chemical Reagent (Beijing, China). Deionized water was prepared through a water purification system (Milli-Q, Milford, MA, USA). Macroporous resins of HPD100 and AB-8 were purchased from CangZhou Bonchem Co., Ltd. (Hebei, China) and Zhengzhou Qinshi Technology (Guangdong, China), respectively. Polyamide resin (30-60 mesh) was supplied by Taizhou Luqiao Sijia Biochemical Plastics Factory (Zhejiang, China). The resins including AMBERLITE FPA98Cl, AMBERLITE FPA90Cl, AMBERLITE FPA53, AMBERLITE XAD 1600N, DOWEX $50 \mathrm{~W} \times 2$, DOWEX $50 \mathrm{~W} \times 4$, and AMBERCHROW CG161M were obtained from Dow Chemical Company (Midland, MI, USA).

\subsection{Optimization of Crude $S A B$ Preparation}

\subsubsection{Procedures}

Deionized water was added to $150 \mathrm{~g}$ of the Danshen under mechanical stirring. The water extract was then filtered and concentrated under reduced pressure. After a small volume of $5 \mathrm{M}$ $\mathrm{HCl}$ solution was added, the liquid-liquid extraction was carried out by contacting the concentrated extract of Danshen with 1-butanol. The $\mathrm{pH}$ values were monitored by a $\mathrm{pH}$ meter (S40 SevenMulti, Mettler-Toledo $\mathrm{GmbH}$, Greifensee, Switzerland) equipped with a combination $\mathrm{pH}$ electrode (InLab Expert Pro, Mettler-Toledo GmbH, Greifensee, Switzerland). After magnetic stirring (85-1, Hangzhou Instrument Motor Co., Ltd., Hangzhou, Zhejiang, China) for $0.5 \mathrm{~h}$, the mixture was centrifuged for $10 \mathrm{~min}$ at a rotation speed of $3000 \mathrm{rpm}$ using a centrifuge (5810R, Eppendorf, Westbury, NY, USA) to separate the two phases. The upper phase was collected and named as Organic Phase 1. Organic Phase 1 was then contacted with deionized water in a separatory funnel. After vigorously shaking, the mixture then was centrifuged for $10 \mathrm{~min}$ at a rotation speed of $3000 \mathrm{rpm}$ to separate two phases. The upper phase was collected again and named as Organic Phase 2. After Organic Phase 2 was collected into a conical flask, deionized water and a small volume of $4 \% \mathrm{NaOH}(w / w)$ solution were added. The mixture was then magnetically stirred for $0.5 \mathrm{~h}$, and then centrifuged. Finally, the aqueous phase was collected and considered to be the crude SAB solution. The schematic diagram of crude SAB preparation is presented in Figure 1.

The crude SAB solution was further purified using a chromatography process. Adsorbents were selected. The elution process was also optimized. The flow-through fractions were concentrated under reduced pressure at $30{ }^{\circ} \mathrm{C}$. Finally, SAB disodium salt was obtained by freeze-drying (MICROMODULYO230, Thermo Electron Corporation, Walthan, MA, USA). The schematic diagram of chromatographic processes and drying were also shown in Figure 1.

\subsubsection{Experimental Design}

To screen significant variables in the preparation of crude SAB, Plackett-Burman-designed experiments were carried out with the conditions displayed in Table 1. Seven parameters were investigated, including extraction temperature, extraction $\mathrm{pH}$, back-extraction $\mathrm{pH}$, extraction time, water consumption for extraction, 1-butanol amount, and water consumption for washing. Box-Behnken designed experiments were used to investigate the relationships between process indices and key parameters of crude SAB preparation process, as shown in Table 2.

\subsection{Optimization of Chromatography}

\subsubsection{Selection of Resins}

Adsorbents

Resins were weighed, then put into a drying oven, and dried at $60{ }^{\circ} \mathrm{C}$ to a constant weight. After that, their moisture was calculated. The physical properties of the resins are given in Table S3. The resins of polyamide resin, HPD100, AB-8, CG161M, and XAD 1600N were washed with ethanol and 
deionized water sequentially before used. The resins, including FPA98Cl, FPA90Cl, $50 \mathrm{~W} \times 2$, and $50 \mathrm{~W} \times 4$, were pretreated using $1 \mathrm{~mol} / \mathrm{L} \mathrm{HCl}, 1 \mathrm{~mol} / \mathrm{L} \mathrm{NaOH}, 1 \mathrm{~mol} / \mathrm{L} \mathrm{HCl}$, and water. FPA53 were pretreated using $1 \mathrm{~mol} / \mathrm{L} \mathrm{HCl}, 1 \mathrm{~mol} / \mathrm{L} \mathrm{NaOH}$, and water in a sequential manner.

\section{Static Adsorption and Desorption Tests}

The crude SAB solution was diluted 10 times $(w / w)$ with deionized water. Resins of $1 \mathrm{~g}$ dry weight and $25 \mathrm{~mL}$ diluted crude SAB solution were added into a $50 \mathrm{~mL}$ conical flask. The flasks were placed in a temperature-controlled oscillator (DSHZ-300, Taicang City Experimental Equipment Factory, Jiangsu, China), shaken at $140 \mathrm{rpm}$ at $30{ }^{\circ} \mathrm{C}$ for $20 \mathrm{~h}$. After adsorption, the resins were desorbed with $40 \mathrm{~mL}$ $70 \%$ ethanol $(v / v)$ solution and shaken at $140 \mathrm{rpm}$ at $30^{\circ} \mathrm{C}$ for $10 \mathrm{~h}$. The concentration of SAB in the solutions after adsorption and desorption were sampled and analyzed using HPLC, respectively.

To select the proper resin, the static adsorption/desorption capacity and ratio of desorption were calculated using the Equations (5)-(7) below:

$$
\begin{gathered}
Q_{e}=\frac{\left(C_{i}-C_{e}\right) V_{i}}{W} \\
Q_{d}=\frac{C_{d} V_{d}}{W} \\
R_{d}=\frac{C_{d} V_{d}}{\left(C_{i}-C_{e}\right) V_{i}} \times 100 \%
\end{gathered}
$$

where $Q_{e}\left(\mathrm{mg} / \mathrm{g}\right.$ dry resin) represents the adsorption capacity under equilibrium; $Q_{d}(\mathrm{mg} / \mathrm{g}$ dry resin) represents the desorption capacity; $R_{d}(\%)$ represents the desorption ratio; $C_{i}$ and $C_{e}(\mathrm{mg} / \mathrm{mL})$ represent the initial and equilibrium concentration of $\mathrm{SAB}$ in the solution, respectively; $V_{i}(\mathrm{~mL})$ represents the volume of the initial solution; $W(\mathrm{~g})$ represents the dry weight of the tested resins; $C_{d}(\mathrm{mg} / \mathrm{mL})$ represents the concentration of $\mathrm{SAB}$ in the desorption solution; and $V_{d}(\mathrm{~mL})$ represents the volume of the desorption solution.

\subsubsection{Adsorption Kinetics Tests}

The crude SAB solution was diluted 50 times $(w / w)$ with deionized water. After that, resins of $1 \mathrm{~g}$ dry weight and $35 \mathrm{~mL}$ dilution were added into a $50 \mathrm{~mL}$ conical flask. The flasks were placed in a temperature-controlled oscillator and shaken at $140 \mathrm{rpm}$ at $30^{\circ} \mathrm{C}$ for $2 \mathrm{~h}$. A total of $0.2 \mathrm{~mL}$ of solution was sampled at 10, 20,30,50,80, and $120 \mathrm{~min}$ to measure the SAB concentration. In order to evaluate the effect of contact time on the adsorption kinetics, the pseudo-second-order model [23-25] was used as followed:

$$
\frac{t}{Q_{t}}=\frac{1}{k_{2} Q_{e}^{2}}+\frac{t}{Q_{e}}
$$

where $Q_{t}$ (mg/g dry resin) is the amount of SAB adsorbed per gram of dry resin at time $t$, and $k_{2}$ is the rate constant of pseudo-second-order kinetics model.

\subsubsection{Optimization of the Chromatographic Process}

Crude SAB solution was diluted five times $(w / w)$ with deionized water. Adsorption of SAB in the diluted crude $\mathrm{SAB}$ solution was carried out in a glass column $(450 \mathrm{~mm} \times 60 \mathrm{~mm})$ packed with the optimized resin. After being washed with $90 \% v / v$ ethanol solution and equilibrated with deionized water, the column was loaded with diluted crude SAB solution at a constant flow rate of $1.94 \mathrm{BV} / \mathrm{h}$. The column was then desorbed with 2 BV deionized water, $10 \% v / v$ ethanol, 30\% v/v ethanol, and $50 \%$ $v / v$ ethanol sequentially at a constant flow rate of $1.94 \mathrm{BV} / \mathrm{h}$. The flow-through fractions were collected at intervals. The SAB concentrations and dry matter contents of the samples were determined. The eluting solvent of chromatographic process and the collection conditions were optimized according to experimental results. 


\subsection{Standard Establishment of the Water Extract}

The medicinal slices of Danshen from five different producing areas were processed to obtain SAB disodium salt. A total of $450.0 \mathrm{~g}$ medicinal slices of Danshen were extracted in $3600 \mathrm{~mL}$ of water under mechanical stirring at $69^{\circ} \mathrm{C}$ for $2.0 \mathrm{~h}$. The water extraction process was repeated once more under the same conditions. The extracts were combined, filtered, and then concentrated at $63{ }^{\circ} \mathrm{C}$ to $1800 \mathrm{~mL}$. The concentrated extract was acidified using $\mathrm{HCl}$ solution to a $\mathrm{pH}$ value of 2.70 . After that, the concentrated extract was contacted with 1-butanol. Then Organic Phase 1 was collected. After washing with water, Organic Phase 2 was collected. Organic Phase 2 was mixed with deionized water, and then basified using a $\mathrm{NaOH}$ solution to a $\mathrm{pH}$ value of 5.00 . The lower phase was collected as a crude SAB solution. Crude SAB solution was diluted five times $(w / w)$ with deionized water. The diluted crude SAB solution was then loaded to the resin column at a constant flow rate of $1.0 \mathrm{BV} / \mathrm{h}$, and then desorbed with deionized water. The flow-through fractions were collected at intervals. After that, the collected flow-through fractions were dried, and finally, the SAB disodium salt was obtained. Seven phenolic compound yield values and purity values were measured to represent water extract features. The SAB yield value and purity value in the final product were also determined. The relationships between water extract features and SAB disodium salt indices were analyzed with the stepwise regression. After that, the quality standards of the water extract were obtained. A new batch of Danshen was extracted and purified to verify the water extract quality standards.

\subsection{Analytical Methods}

The quantitative measurement of DSS, PA, CA, RA, LA, SAB, and SAA were carried out via HPLC analysis according to the method published by Cao et al. [4]. Two HPLC systems were used, including HP 1100 series (Agilent Technologies, Palo Alto, CA, USA) was equipped with Chemstation software (version number: B.04.03, Agilent Technologies), and LC5090 series (Zhejiang Fuli analytical instrument Co., Ltd., Wenling, China) was equipped with a Chemstation software (version number: 1.5.0.0, Zhejiang Fuli analytical instrument Co., Ltd.). The separations were performed on an Agilent Zorbax Extend reversed-phase C18 column $(5 \mu \mathrm{m}, 250 \times 4.6 \mathrm{~mm})$ purchased from Agilent (Santa Clara, CA, USA). The flow rate, the injection volume, and the column oven temperature were $1.0 \mathrm{~mL} / \mathrm{min}$, $2 \mathrm{~mL}$, and $25^{\circ} \mathrm{C}$, respectively. The detection wavelength was $281 \mathrm{~nm}$. The mobile phase consisted of $0.1 \%(v / v)$ formic acid aqueous solution (A) and acetonitrile $(\mathrm{B})$ with the gradient program as follows: $0-10 \mathrm{~min}, 7-17 \%$ of $\mathrm{B} ; 10-16 \mathrm{~min}, 17-21 \%$ of $\mathrm{B} ; 16-31 \mathrm{~min}, 21-21 \%$ of $\mathrm{B} ; 32-40 \mathrm{~min}, 21-29 \%$ of $\mathrm{B} ; 40-44 \mathrm{~min}, 29-35 \%$ of $\mathrm{B} ; 44-45 \mathrm{~min}, 35-100 \%$ of $\mathrm{B}$. The dry matter contents were determined gravimetrically using hot air drying at $105{ }^{\circ} \mathrm{C}$ to a constant weight [26].

\subsection{Data Analysis}

The purity of phenolic compounds $\left(P C_{\text {Purity }}\right)$, including DSS, PA, CA, RA, LA, SAB, and SAA, were calculated using Equations (9) and (10):

$$
\begin{aligned}
& P C_{\text {Purity }}^{E}=C_{i}^{E} M_{i}^{E} / D M^{E} \times 100 \%(i=1,2 \ldots, 7) \\
& P C_{\text {Purity }}^{S}=C_{i}^{S} M_{i}^{S} / D M^{S} \times 100 \%(i=1,2 \ldots, 7)
\end{aligned}
$$

where $C$ and $M$ refer to the concentration and mass, respectively; $D M$ is the mass of dry matter; superscript $E$ and $S$ are the water extract and crude $S A B$ solution, respectively; and subscript $i$ refers to DSS, PA, CA, RA, LA, SAB, and SAA, respectively.

The yield of phenolic compounds $\left(P C_{\text {yield }}\right)$ were defined as in Equations (11) and (12):

$$
P C_{\text {yield }}^{E}=\frac{C_{i}^{E} M_{i}^{E}}{M_{D S}}
$$




$$
P C_{\text {yield }}^{S}=\frac{C_{i}^{S} M_{i}^{S}}{M_{D S}}
$$

where $M_{D S}$ is the mass of the Danshen.

$\mathrm{SAB}$ yield in crude $\mathrm{SAB}$ solution $\left(S A B_{\text {yield }}^{S}\right)$ and $\mathrm{SAB}$ purity in crude $\mathrm{SAB}$ solution $\left(S A B_{\text {purity }}^{S}\right)$ was considered as the process indices for the preparation processes of crude $S A B$. To reflect the comprehensive effects of process parameters on all evaluation indicators, the key parameters of crude SAB preparation process were identified by employing the method of the standard partial regression coefficient [27-29]. The response variables of $Y_{1}$ and $Y_{2}$ were standardized firstly according to following Equation (13). After that, the standard partial regression coefficients were calculated by multivariate linear regression based on the Equation (14). Finally, the absolute values of each standard partial regression coefficient were weighted and summed up to get $I F$ values according to Equation (15). Parameters with higher $I F$ values were expected to have greater influences on responses.

$$
\begin{gathered}
Y_{k}^{\prime}=\frac{Y_{k}-\bar{Y}_{k}}{S T D_{k}}(k=1,2) \\
Y_{k}^{\prime}=\beta_{0, k}+\sum_{j=1}^{7} \beta_{j, k} X_{j}(j=1,2, \ldots, 7) \\
I F_{j}=\sum_{k=1}^{2} w_{k}\left|\beta_{j, k}\right|
\end{gathered}
$$

where $Y_{k}$ is the measured value; $Y_{k}^{\prime}$ and $\bar{Y}_{k}$ are the standardized value and average value of each response, respectively; $S T D_{k}$ is the standard deviation of each response; and number $k(k=1,2)$ represents $S A B_{\text {yield }}^{S}$ and $S A B_{\text {purity }}^{S}$ in crude $S A B$ solution. $\beta_{0, k}$ is a constant term, $X_{j}$ is the coded value of a parameter, subscript $j$ refers to a process parameter, and $\beta_{j, k}$ is the standard partial regression coefficient. In this study, each response was considered equally important, which signifies that the $w_{k}$ values were both $1 / 2$.

The data of Box-Behnken-designed experiments were modeled with Equation (16) in terms of the coded factors below:

$$
Y=a_{0}+\sum_{i=1}^{3} a_{i} X_{i}+\sum_{i=1}^{3} a_{i i} X_{i}^{2}+\sum_{j=i+1}^{3} a_{i j} X_{i} X_{j}(i=1,2,3 ; j=2,3)
$$

where $a_{0}$ is a constant; $a_{i}, a_{i i}$, and $a_{i j}$ are the regression coefficients for the linear, quadratic, and interaction of the model, respectively. The meaning of variable $X$ in Equation (14) and Equation (16) was the same. Stepwise regression was carried out to establish quantitative models between process indices and key parameters of crude SAB preparation process. The significance level for moving in or out a term was set to 0.1. The Design Expert 8.0.6 software (Stat-Ease Inc., Minneapolis, MN, USA) was used for the calculations.

\section{Conclusions}

In this work, SAB disodium salt was successfully prepared from Danshen through a series of processes including water extraction, concentration, acidification, 1-butanol extraction, water washing, basification, water back extraction, chromatography, and drying. Three parameters of extraction temperature, extraction $\mathrm{pH}$, and back-extraction $\mathrm{pH}$ were found to be significantly affect the $\mathrm{SAB}$ purity and yield of the crude SAB solution. The optimized ranges of these parameters were given. CG161M resin was selected for further purification of crude SAB solution due to its high adsorption capacity, high desorption ratio, and fast adsorption kinetics. The conditions of chromatography were also optimized. Water was used as the eluent for the purification of SAB disodium salt. It is low cost and environment-friendly. About $5 \mathrm{~g}$ of high-purity SAB disodium salt $(>95 \%)$ could be obtained in 
one preparation process. The productivity of the present method was larger compared with other reported works.

Different batches of Danshen were used to prepare SAB disodium salt with the optimized parameters. Water extract indices were considered to represent the effects of Danshen quality and water extraction process. Therefore, phenolic compound purity and phenolic compound yield of water extracts were measured. After the quantitative relationships between SAB disodium salt purity and yield and water extract indices were established, the quality standard of Danshen water extract was calculated and verified. With the setting of intermediate quality standards, product quality can be predicted at an early stage of manufacturing processes. The method used in this work can also be applied to develop process intermediate quality standards for other natural products.

Supplementary Materials: Supplementary materials are available online at http:/ /www.mdpi.com/1420-3049/ 24/7/1269/s1. Table S1: Partial regression coefficients, importance factors, and coefficient confidence intervals, Table S2: Estimated parameter values, determination coefficients, ANOVA results, and coefficient confidence intervals of quadratic models, Table S3: Physical properties of the test macroporous resins; Figure S1: FTIR spectrum of SAB disodium salt and SAB, Figure S2: TOF-MS spectrum of SAB disodium salt.

Author Contributions: Conceptualization, H.Q. and X.G.; Data curation, T.Y.; Formal analysis, T.Y.; Funding acquisition, X.G.; Investigation, T.Y.; Project administration, H.Q.; Resources, H.Q. and X.G.; Software, X.G.; Supervision, H.Q.; Writing—original draft, T.Y.; Writing—review and editing, H.Q. and X.G.

Funding: This work was supported by the National Natural Science Foundation of China (No. 81503242) and the Fundamental Research Funds for the Central Universities (No. 2018FZA7018).

Acknowledgments: We thank Li Ma, Hongbin Zou, Zhiheng Shu, Shufang Wang, and Zhiwei Ge for their help during the identification of SAB disodium salt.

Conflicts of Interest: The authors declare no conflicts of interest.

\section{References}

1. Liang, W.Y.; Chen, W.J.; Wu, L.F.; Li, S.; Qi, Q.; Cui, Y.P.; Liang, L.J.; Ye, T.; Zhang, L.Z. Quality evaluation and chemical markers screening of salvia miltiorrhiza Bge. (Danshen) based on HPLC fingerprints and HPLC-MSn coupled with chemometrics. Molecules 2017, 22, 478. [CrossRef]

2. Liu, A.H.; Li, L.; Xu, M.; Lin, Y.H.; Guo, H.Z.; Guo, D.A. Simultaneous quantification of six major phenolic acids in the roots of Salvia miltiorrhiza and four related traditional Chinese medicinal preparations by HPLC-DAD method. J. Pharm. Biomed. Anal. 2006, 41, 48-56. [CrossRef] [PubMed]

3. Zeng, H.T.; Su, S.L.; Xiang, X.; Sha, X.X.; Zhu, Z.H.; Wang, Y.Y.; Guo, S.; Yan, H.; Qian, D.W.; Duan, J.N. Comparative Analysis of the Major Chemical Constituents in Salvia miltiorrhiza Roots, Stems, Leaves and Flowers during Different Growth Periods by UPLC-TQ-MS/MS and HPLC-ELSD Methods. Molecules 2017, 22, 771. [CrossRef] [PubMed]

4. Cao, J.; Wei, Y.J.; Qi, L.W.; Li, P.; Qian, Z.M.; Luo, H.W.; Chen, J.; Zhao, J. Determination of fifteen bioactive components in Radix et Rhizoma Salviae Miltiorrhizae by high-performance liquid chromatography with ultraviolet and mass spectrometric detection. Biomed. Chromatogr. 2008, 22, 164-172. [CrossRef] [PubMed]

5. Shi, M.; Sun, W.Y.; Sun, G.X.; Zhang, S.J. Total monitoring of the constituents of Danshen tablet using micellar electrokinetic chromatography fingerprinting for antioxidant activity profilin. J. Sep. Sci. 2016, 39, 1776-1784. [CrossRef] [PubMed]

6. Wu, X.J.; Wang, Y.P.; Wang, W.; Sun, W.K.; Xu, Y.M. Free radical scavenging and inhibition of lipid peroxidation by magnesium lithospermate B. Acta. Pharmacol. Sin. 2000, 21, 855-858.

7. Tang, M.K.; Ren, D.C.; Zhang, J.T.; Du, G.H. Effect of salvianolic acids from Radix Salviae miltiorrhizae on regional cerebral blood flow and platelet aggregation in rats. Phytomedicine 2002, 9, 405-409. [CrossRef]

8. Yue, J.M.; Li, B.; Jing, Q.P.; Guan, Q.B. Salvianolic acid B accelerated ABCA1-dependent cholesterol efflux by targeting PPAR- $\gamma$ and LXR $\alpha$. Biochem. Bioph. Res. Co. 2015, 462, 233-238. [CrossRef]

9. Gong, X.C.; Wang, S.S.; Qu, H.B. Comparison of two separation technologies applied in the manufacture of botanical injections: Second ethanol precipitation and solvent extraction. Ind. Eng. Chem. Res. 2011, 50, 7542-7548. [CrossRef] 
10. Sun, Y.S.; Zhu, H.F.; Wang, J.H.; Liu, Z.B.; Bi, J.J. Isolation and purification of salvianolic acid A and salvianolic acid $B$ fromSalvia miltiorrhiza by high-speed counter-current chromatography and comparison of their antioxidant activity. J. Chromatogr. B 2009, 877, 733-737. [CrossRef]

11. Ming, G.; Wang, X.L.; Su, Z.G.; Fan, O.Y. One-step separation and purification of 3,4-dihydroxyphenyllactic acid, salvianolic acid B and protocatechualdehyde from Salvia miltiorrhiza Bunge by high-speed counter-current chromatography. J. Chromatogr. A 2007, 1140, 107-111.

12. Chen, J.H.; Wang, F.M.; Lee, F.S.C.; Wang, X.R.; Xie, M.Y. Separation and identification of water-soluble salvianolic acids from Salvia miltiorrhiza Bunge by high-speed counter-current chromatography and ESI-MS analysis. Talanta 2006, 69, 172-179. [CrossRef] [PubMed]

13. LI, H.B.; Lai, J.P.; Jiang, Y.; Chen, F. Preparative isolation and purification of salvianolic acid B from the Chinese medicinal plant Salvia miltiorrhiza by high-speed counter-current chromatography. J. Chromatogr. A 2002, 943, 235-239. [CrossRef]

14. Kan, S.D.; Li, J.A.; Huang, W.Y.; Shao, L.; Chen, D.J. Microsphere resin chromatography combined with microbial biotransformation for the separation and purification of salvianolic acid $\mathrm{B}$ in aqueous extract of roots of Salvia multiorrihza Bunge. J. Chromatogr. A 2009, 1216, 3881-3886. [CrossRef] [PubMed]

15. Guo, Y.X.; Zhou, L.L.; Li, T.; Wang, L.H. Preparative separation of lithospermic acid B from Salvia miltiorrhiza by polyamide resin and preparative high-performance liquid chromatography. J. Chromatogr. A 2011, 1218, 4606-4611. [CrossRef]

16. Xu, J.; Tan, T.W.; Janson, J.C. One-step simultaneous purification of three water-soluble constituents in crude extracts from Danshen by adsorption chromatography on oligo-b-cyclodextrin substituted agarose gel media. Process Biochem. 2007, 42, 480-485. [CrossRef]

17. Nie, L.R.; Lu, J.; Zhang, W.; He, A.; Yao, S. Ionic liquid-modified silica gel as adsorbents for adsorption and separation of water-soluble phenolic acids from Salvia militiorrhiza Bunge. SEP. Purif. Technol. 2015, 155, 2-12. [CrossRef]

18. Guo, Y.X.; Shi, C.Z.; Zhang, L.; Lv, L.; Zhang, Y.Y. Extraction and isolation of lithospermic acid B fromSalvia miltiorrhiza Bunge using aqueous two-phase extraction followed by high-performance liquid chromatography. J. Sep. Sci. 2016, 39, 3624-3630. [CrossRef] [PubMed]

19. Li, Y.; Wang, N.; Zhang, M.; Ito, Y.; Zhang, H.Y.; Wang, Y.R.; Guo, X.; HU, P. Development of a method to extract and purify target compounds from medicinal plants in a single step: Online hyphenation of expanded bed adsorption chromatography and countercurrent chromatography. Anal. Chem. 2014, 86, 3373-3379. [CrossRef] [PubMed]

20. Zhong, W.; Chen, S.; Zhang, J.; Wang, Y.S.; LIu, A. Which one is more important, raw materials or productive technology?-A case study for quality consistency control of Gegen Qinlian decoction. China J. Chin. Mater. Med. 2016, 41, 1027-1032.

21. Chen, T.; Gong, X.C.; Chen, H.L.; Zhang, Y.; Qu, H.B. Chromatographic elution process design space development for the purification of saponins in Panax notoginseng extract using a probability-based approach. J. Sep. Sci. 2016, 39, 306-315. [CrossRef]

22. Ni, L.J.; Gu, H.X.; Gao, X.J.; Zhang, L.G. A new technology of extracting salvianolic acid B from Salviamiltiorrhiza Bunge. J. East China Univ. Sci. Techno.:Nat. Sci. Ed. 2006, 32, 964-966.

23. Darabi, S.F.; Bahramifar, N.; Khalilzadeh, M.A. Equilibrium, thermodynamic and kinetics studies on adsorption of eosin $\mathrm{Y}$ and red $\mathrm{X}-\mathrm{GRL}$ from aqueous solution by treated rice husk. J. App. Res. Wat. Wast. 2018, 9, 392-398.

24. Gao, C.; Zhao, S.M.; Yagiz, Y.; Gu, L.W. Static, kinetic, and isotherm adsorption performances of macroporous adsorbent resins for recovery and enrichment of bioactive procyanidins from cranberry pomace. J. Food Sci. 2018, 83, 1249-1257. [CrossRef] [PubMed]

25. Valderrama, C.; Cortina, J.L.; Farran, A.; Gamisans, X.; Lao, C. Kinetics of sorption of polyaromatic hydrocarbons onto granular activated carbon and Macronet hyper-cross-linked polymers (MN200). J. Colloid Interf. Sci. 2007, 310, 35-46. [CrossRef]

26. Committee, N.P. Pharmacopoeia of the People's Republic of China, 2015 ed.; China medical science and technology press: Beijing, China, 2015; p. 202.

27. Liu, S.Y.; Shen, J.J.; Li, W.L.; Qu, H.B.; Gong, X.C. Comparison on three methods for identification of critical process parameters. Chin. Tradit. Herbal Drugs 2016, 47, 3193-3198. 
28. Yan, B.J.; Guo, Z.T.; Qu, H.B.; Zhao, B.C.; Zhao, T. An approach to determine critical process parameters for ethanol precipitation process of danhong injection. China J. Chin. Mater. Med. 2013, 38, 1672-1675.

29. Shao, J.Y.; Cao, W.; Qu, H.B.; Pan, J.Y.; Gong, X.C. A novel quality by design approach for developing an HPLC method to analyze herbal extracts: A case study of sugar content analysis. PLoS ONE 2018. [CrossRef]

Sample Availability: Samples of the compounds are available from the authors.

(C) 2019 by the authors. Licensee MDPI, Basel, Switzerland. This article is an open access article distributed under the terms and conditions of the Creative Commons Attribution (CC BY) license (http:/ / creativecommons.org/licenses/by/4.0/). 